\title{
DARK MASS MOMENTS
}

\author{
A. De Rújula, Ph. Jetzer and E. Massó
}

CERN, CH-1211 Geneva, Switzerland

\begin{abstract}
In a potentially seminal paper Paczyński has demonstrated a way to detect the "dark" constituents of the halo of our galaxy, should their mass lie in the approximate domain $10^{-6}<M / M_{\odot}<10^{-1}$. (This range snugly and auspiciously overlaps with that of $\mathrm{H} / \mathrm{He}$ aggregates heavy enough not to evaporate, but too light to ignite or to be visible). The massive halo objects (MHOs), when crossing close to the line of sight of a more distant and "fixed" star, would gravitationally "micro-lense" its image, resulting in a time-dependent achromatic amplification with a predictable shape. Observations now in preparation are to monitor up to one million stars in the Large Magellanic Cloud (LMC) during 1 year. Barring unsuspected backgrounds, these observations will either establish or exclude a halo dark mass constituency in the quoted mass range. In the event of a discovery, the next most crucial question will concern the MHO mass distribution, well concealed in the raw data by convolutions with the distributions of various unobservable quantities, that describe the motion and position of individual MHOs. We discuss the extraction from the data of this MHO mass function and we prove that its moments are explicitly related to the moments of the observed distribution of durations of the micro-lensing events.
\end{abstract}

CERN-TH.5812/90

August 1990 


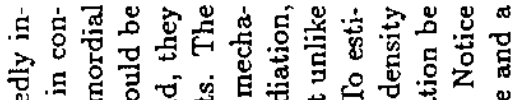

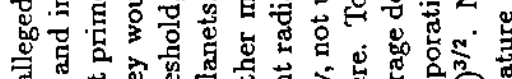

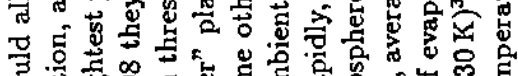

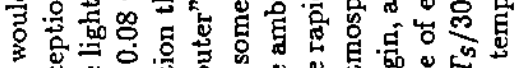

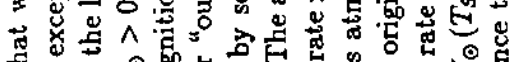

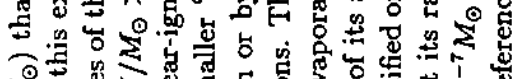

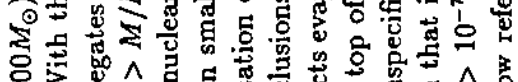
等

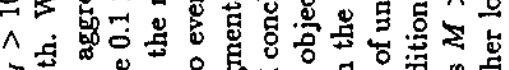

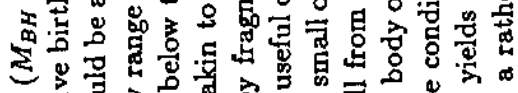

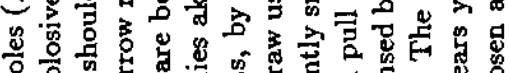

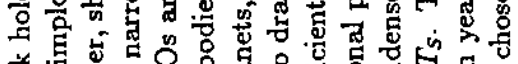

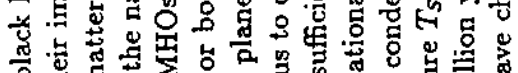

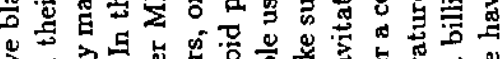

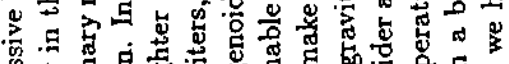

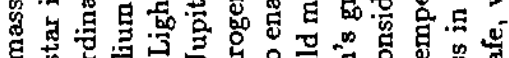

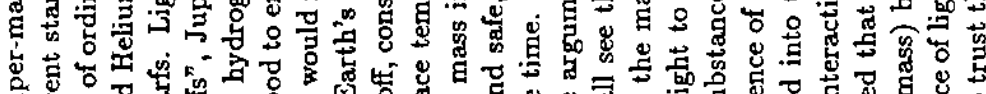

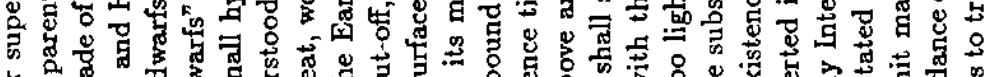

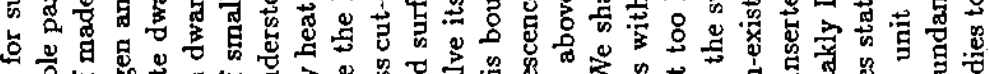

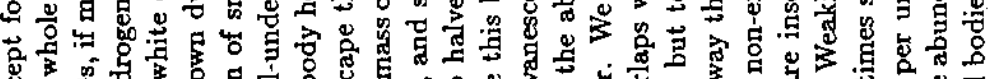

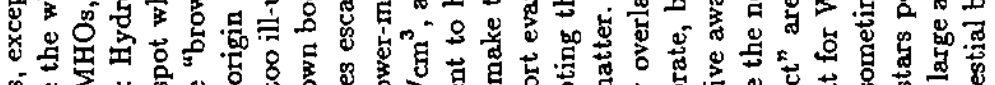

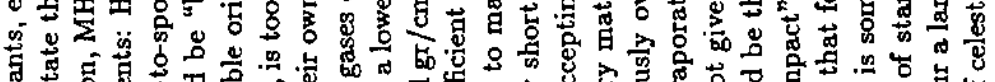

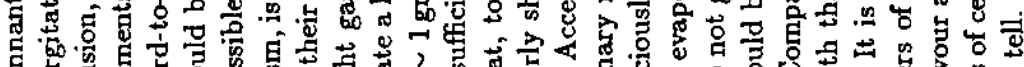

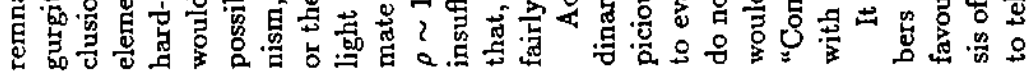

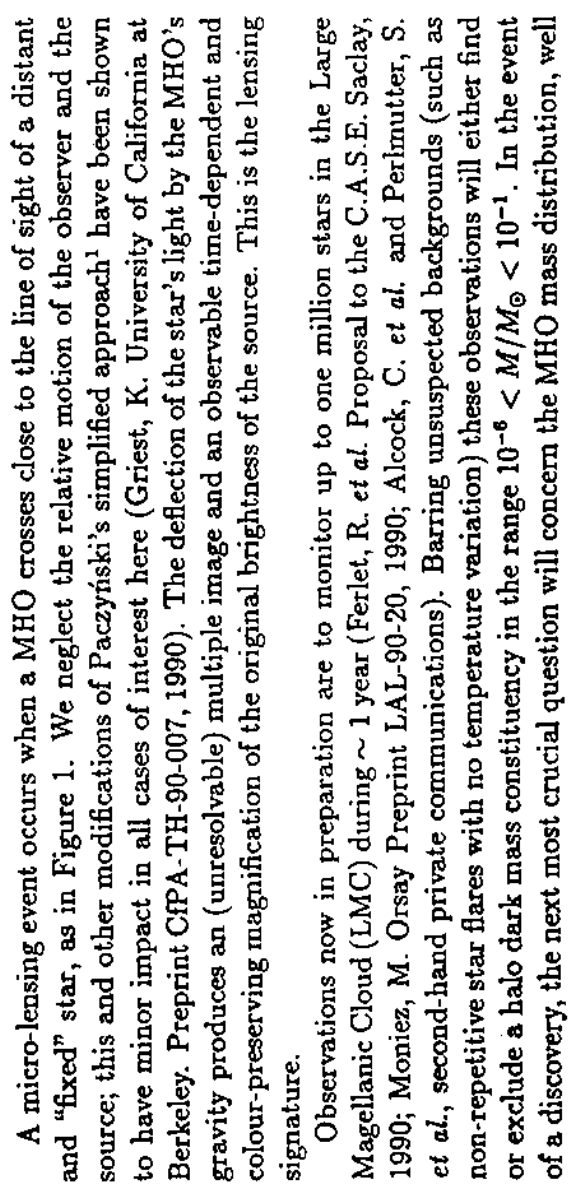

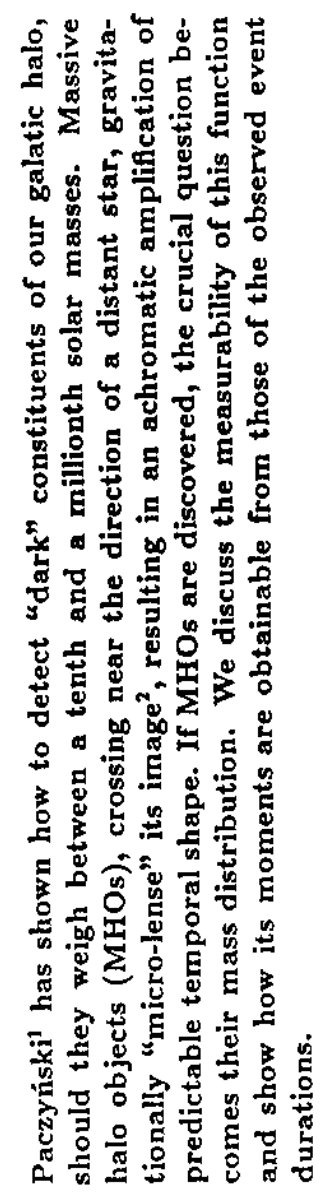

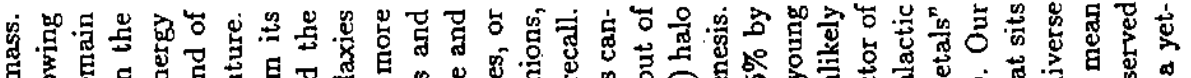

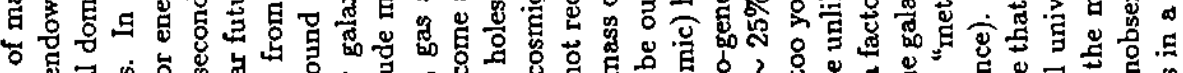

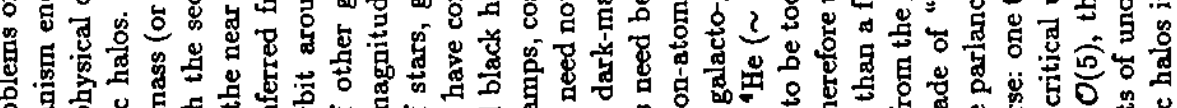

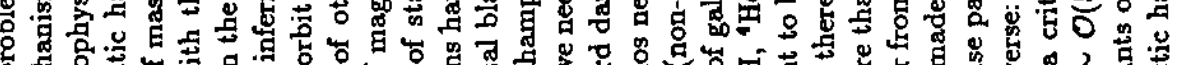

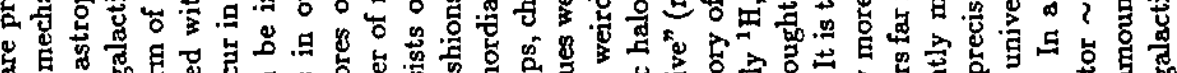

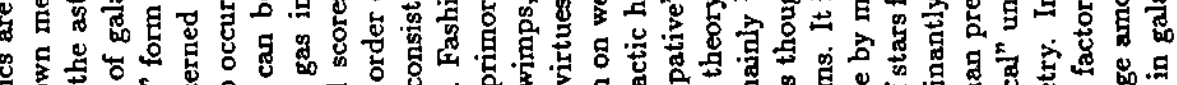

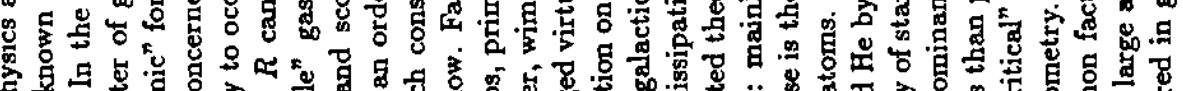

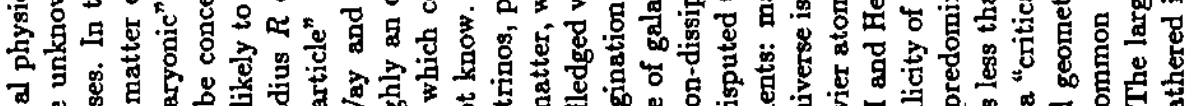

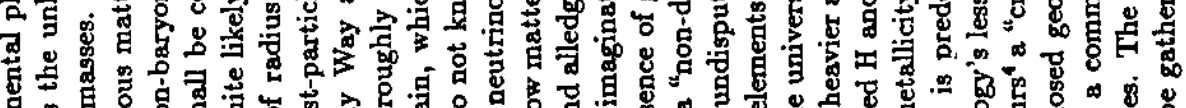

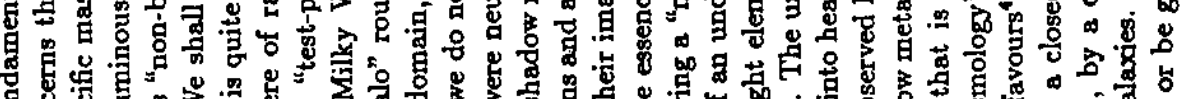

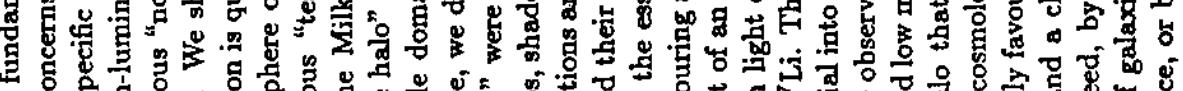

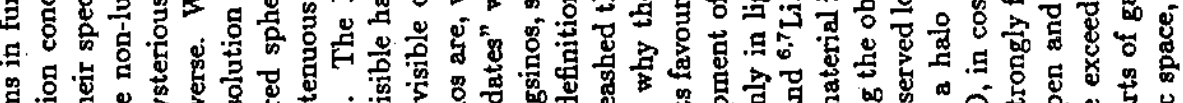

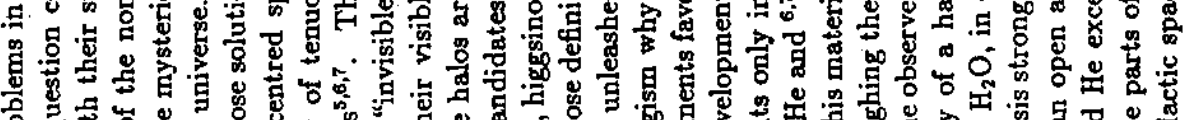

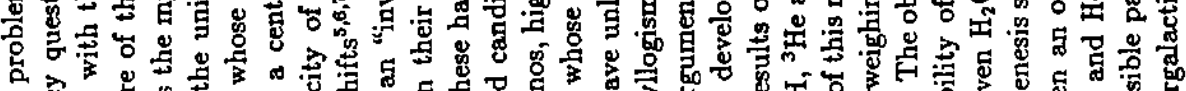

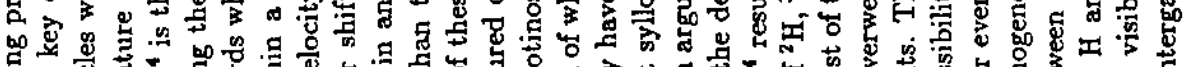

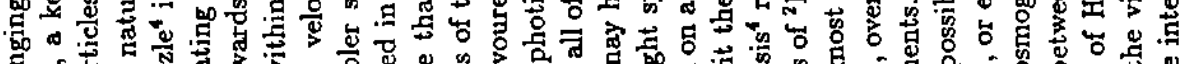

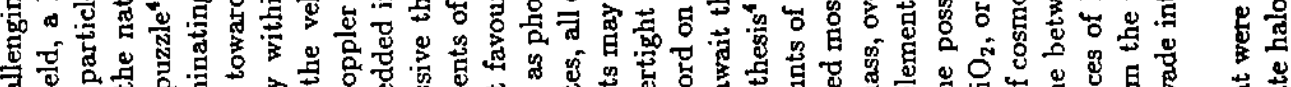

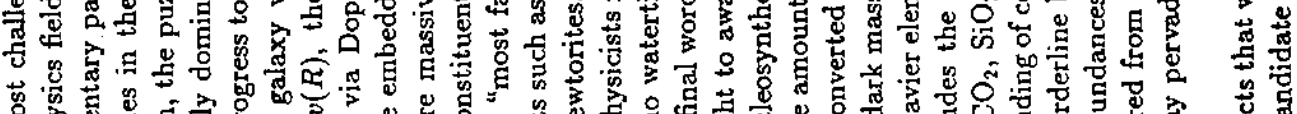

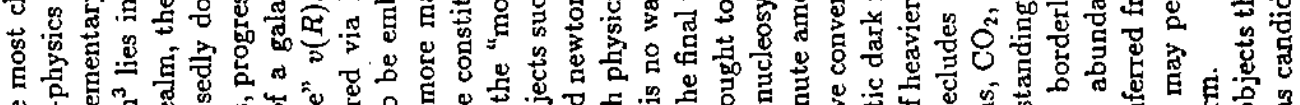

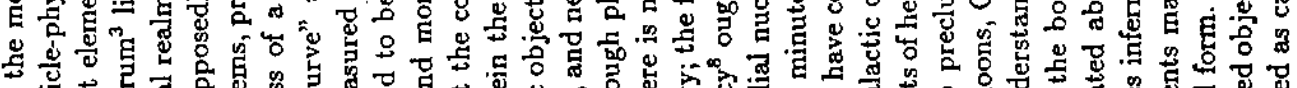

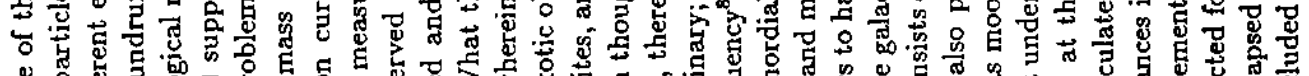

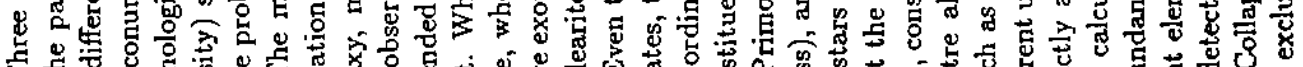

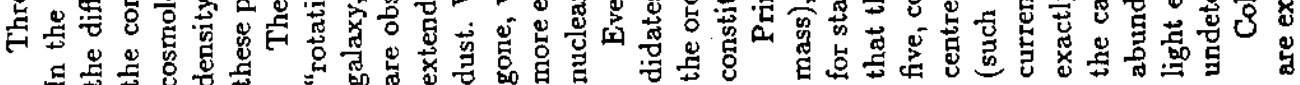



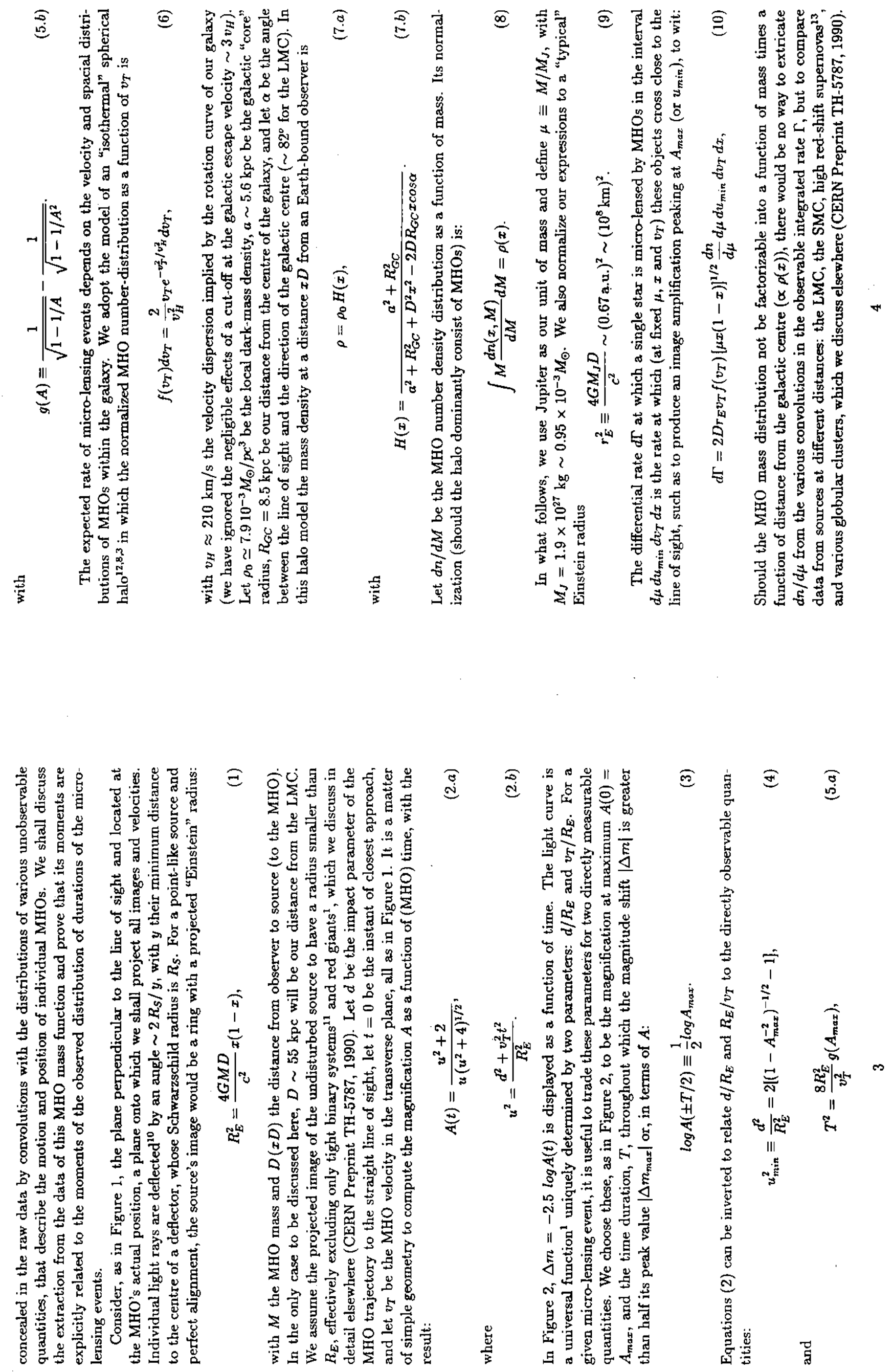

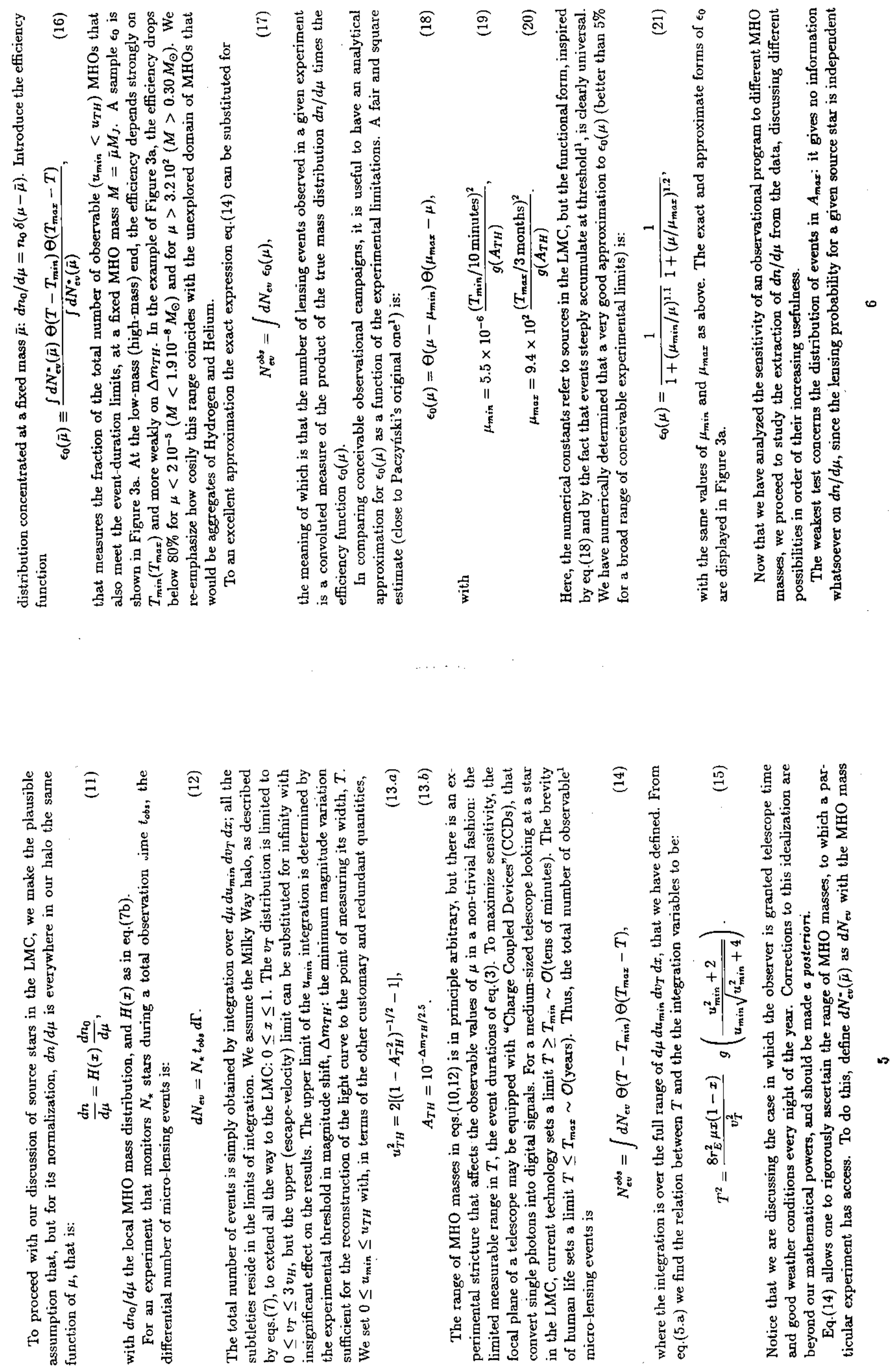

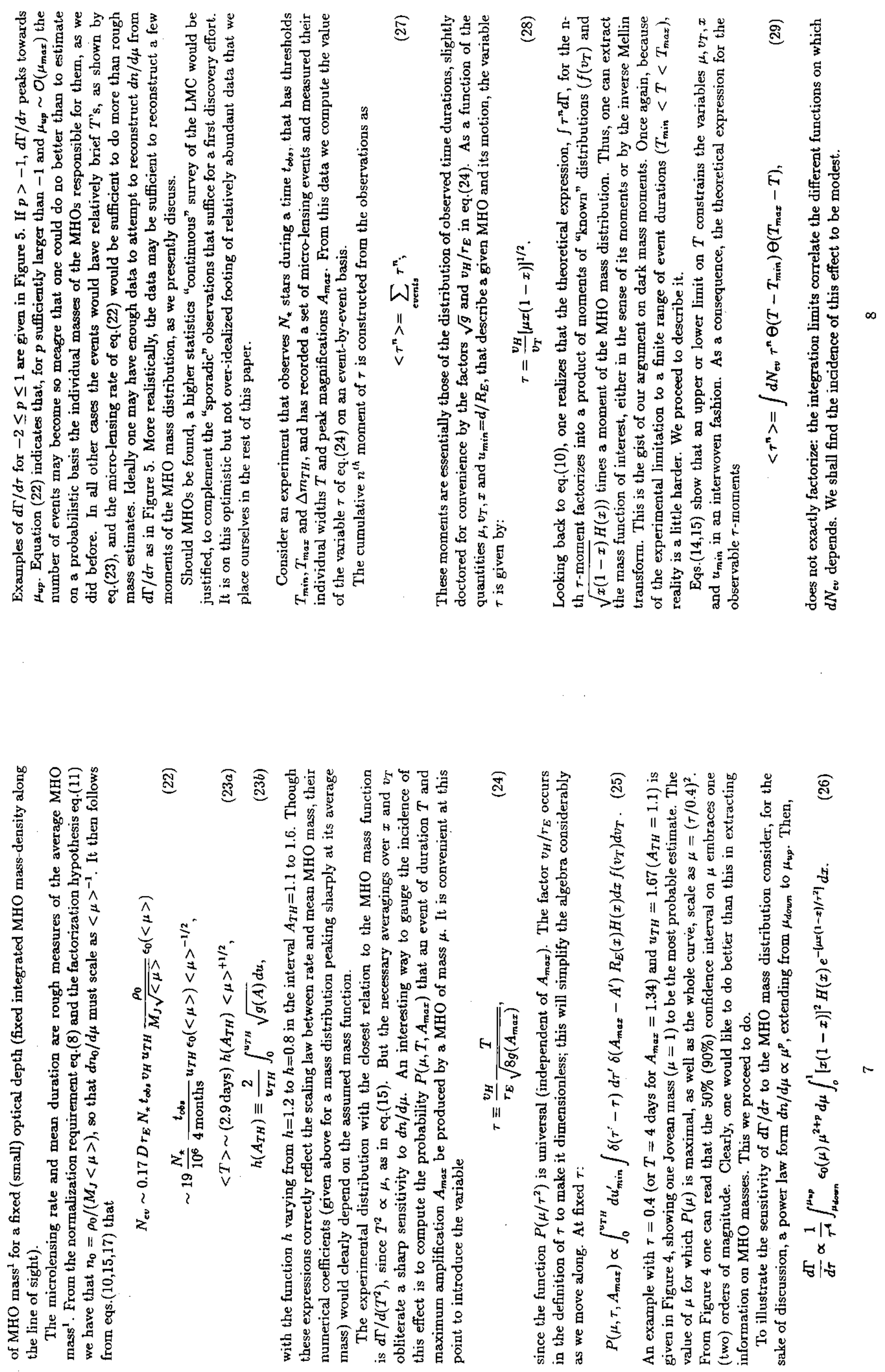


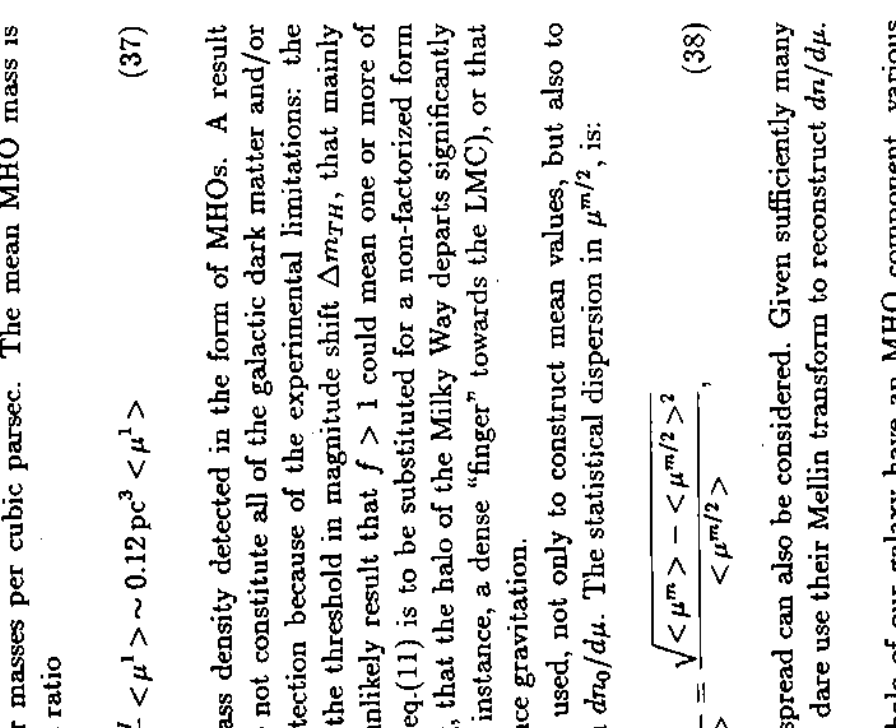

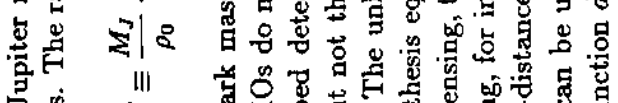

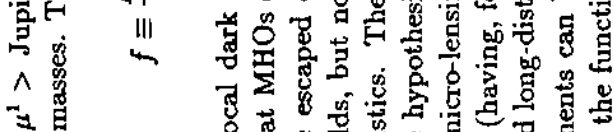

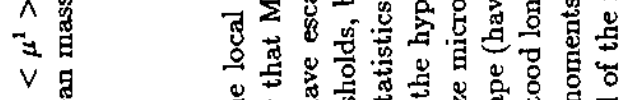

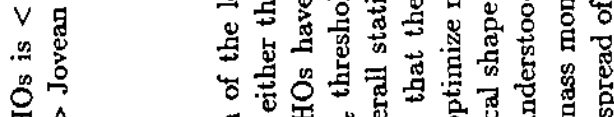

象公

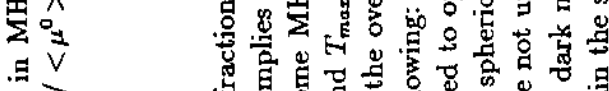

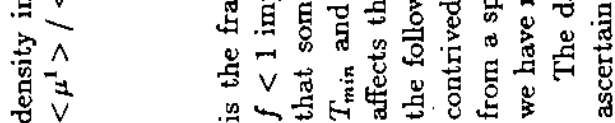

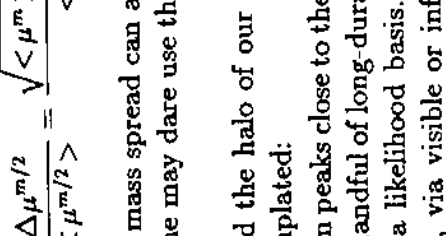

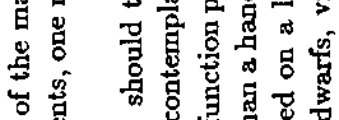

吨

焉

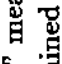

总首

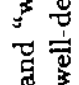
- 4 出

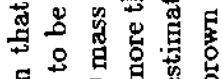

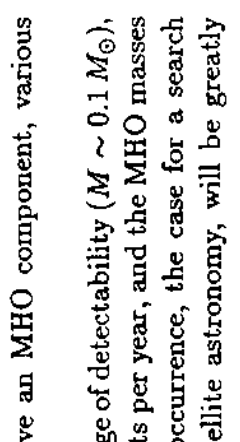

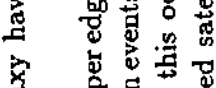

蛋

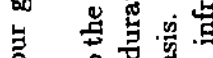

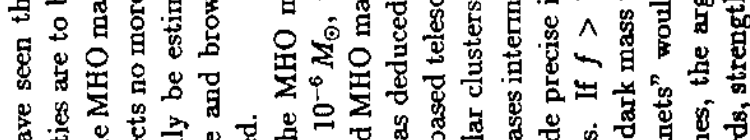

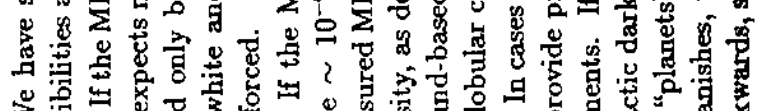

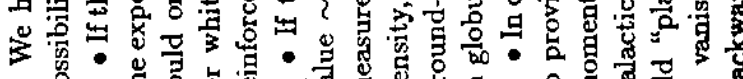

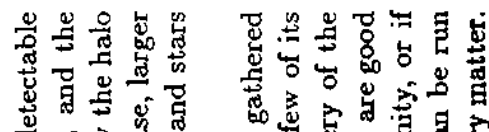

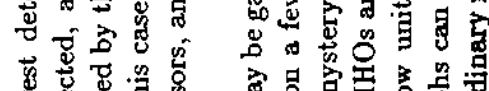

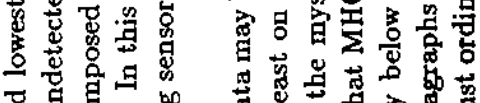

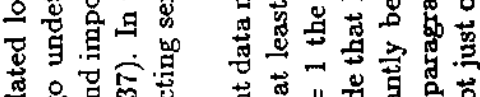

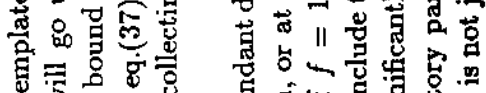

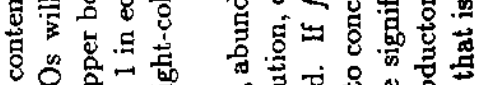

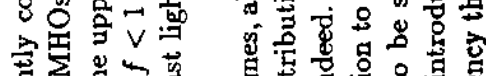

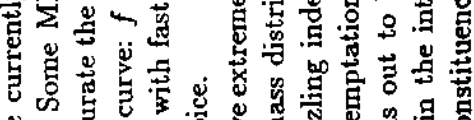

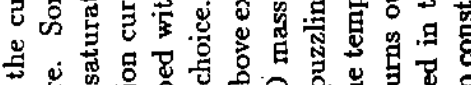

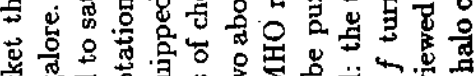

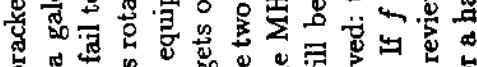

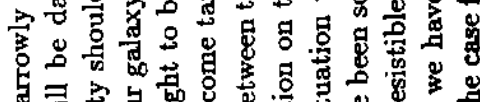

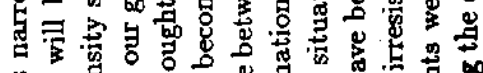

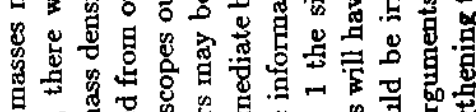
웅

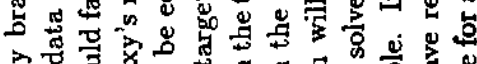

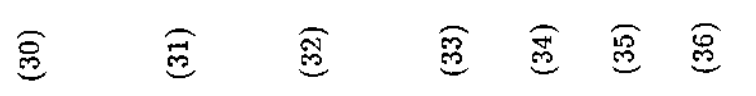

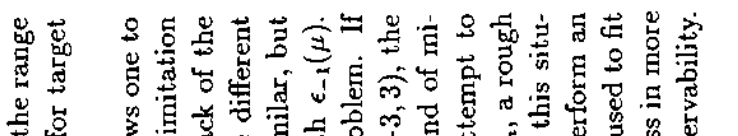

点

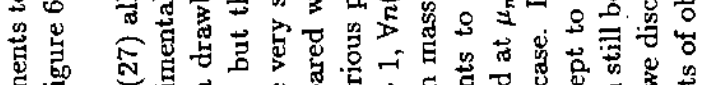

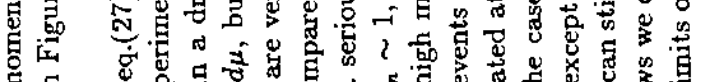

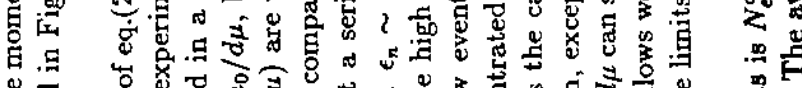

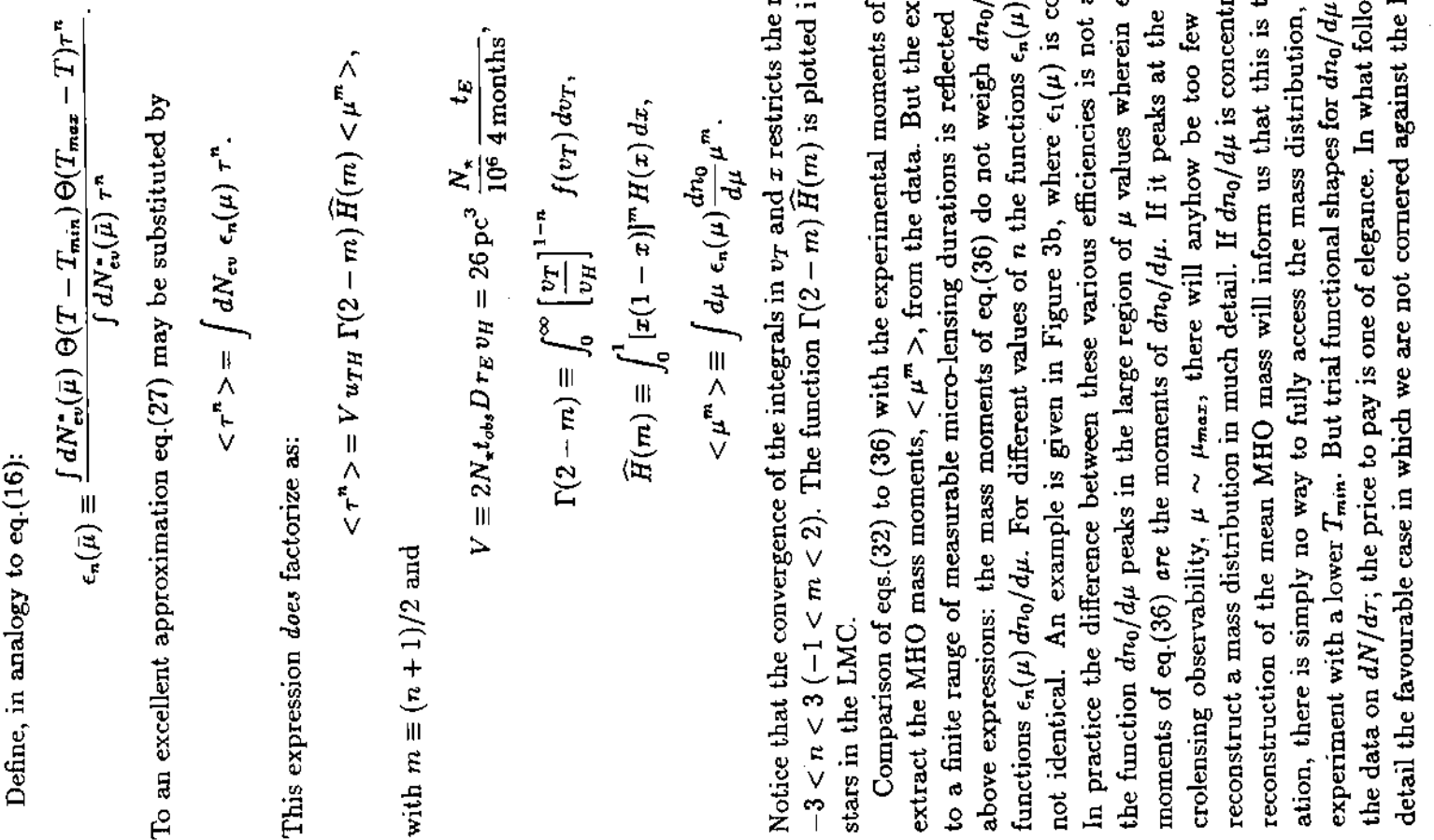



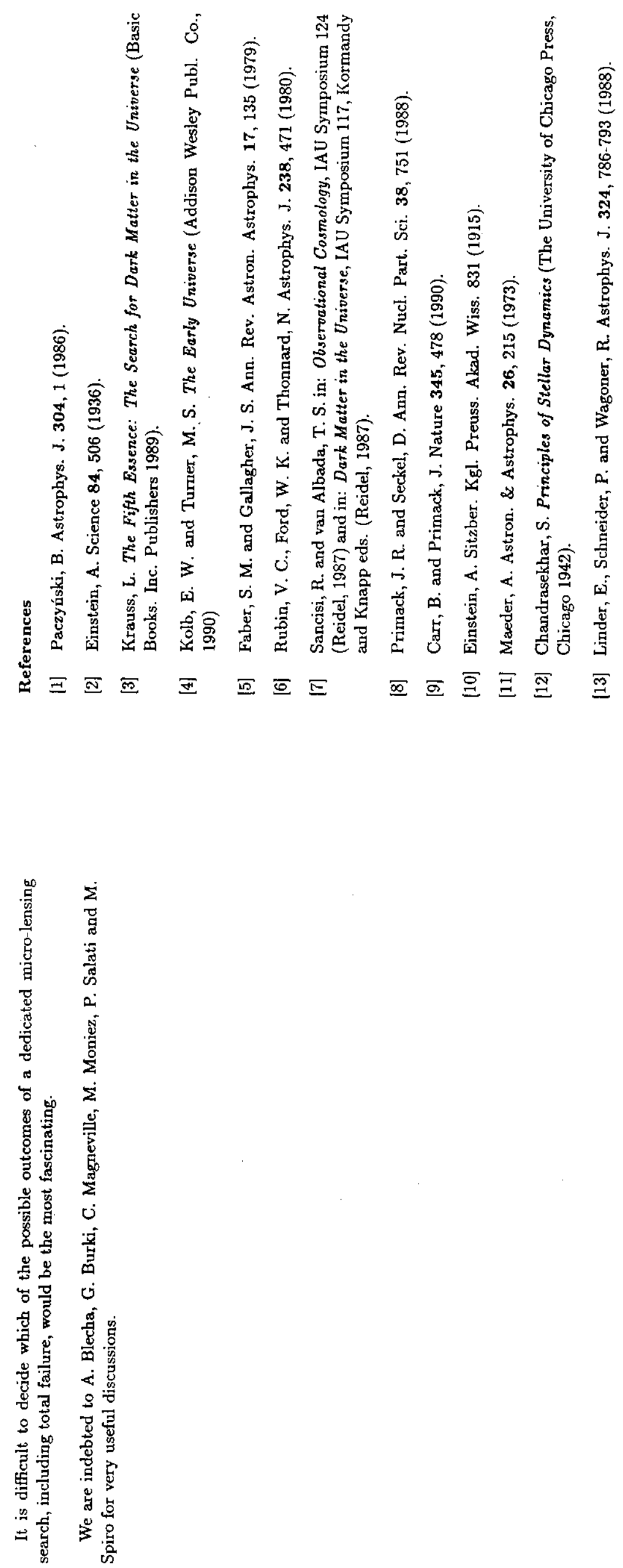

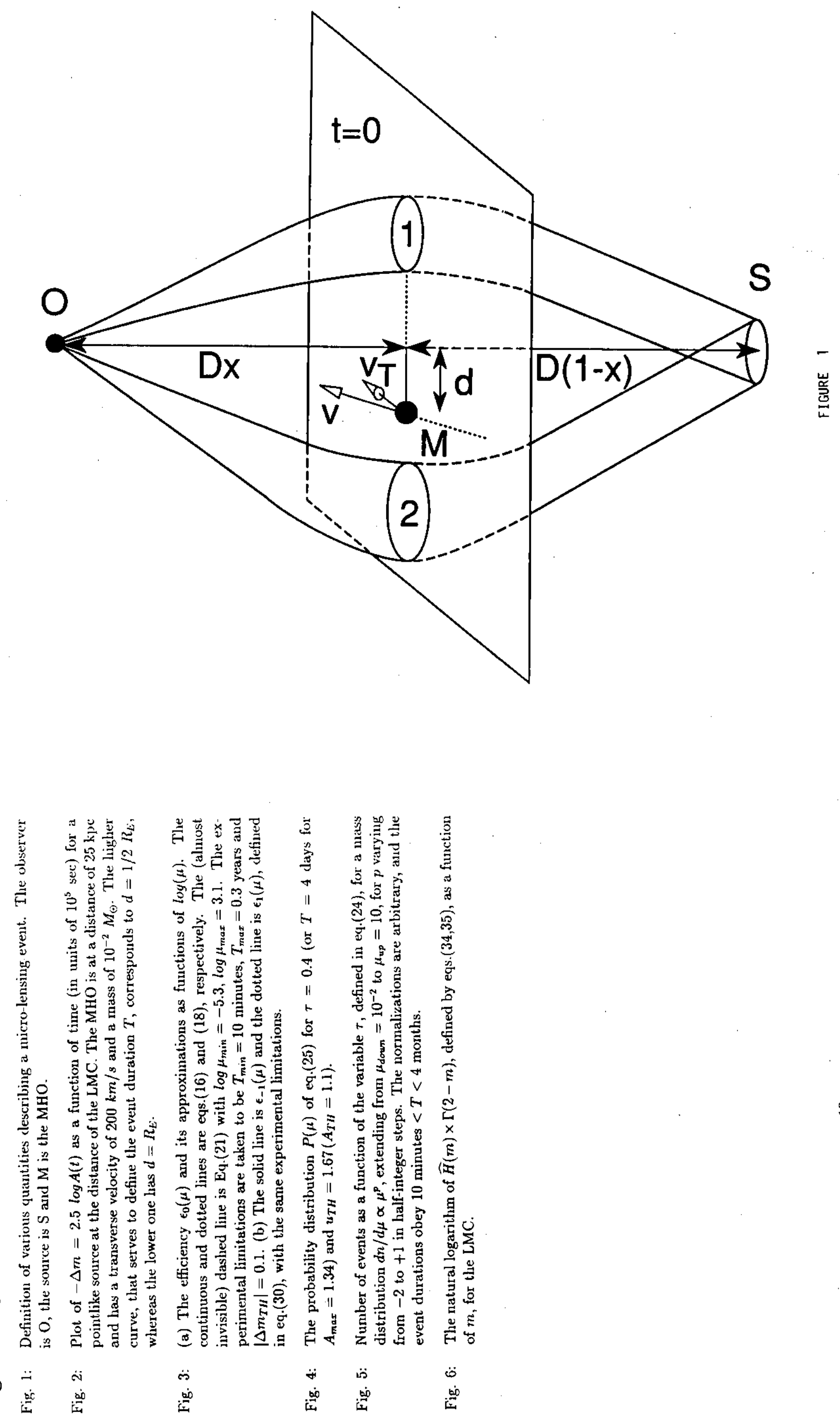


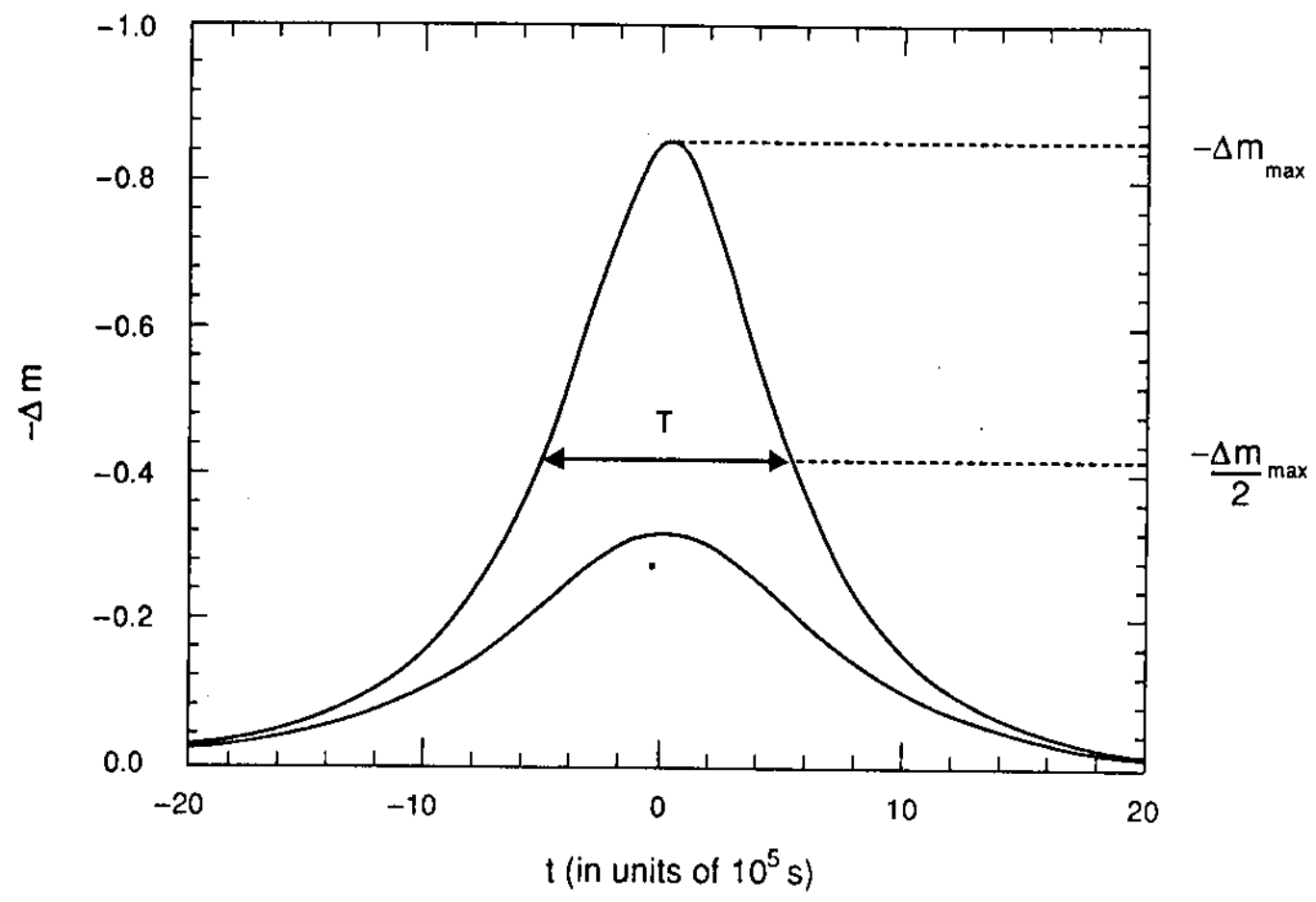

FIGURE 2

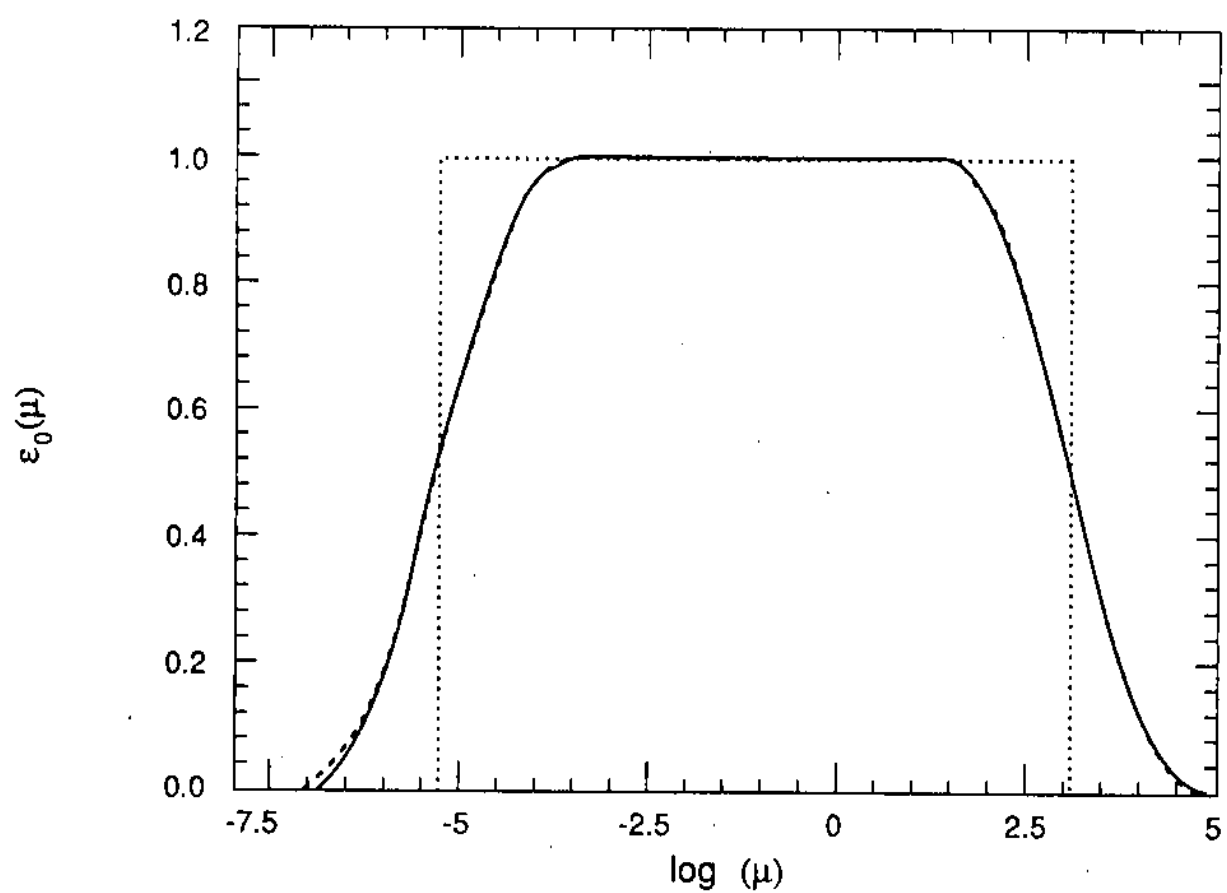

FIGURE $3 a$ 


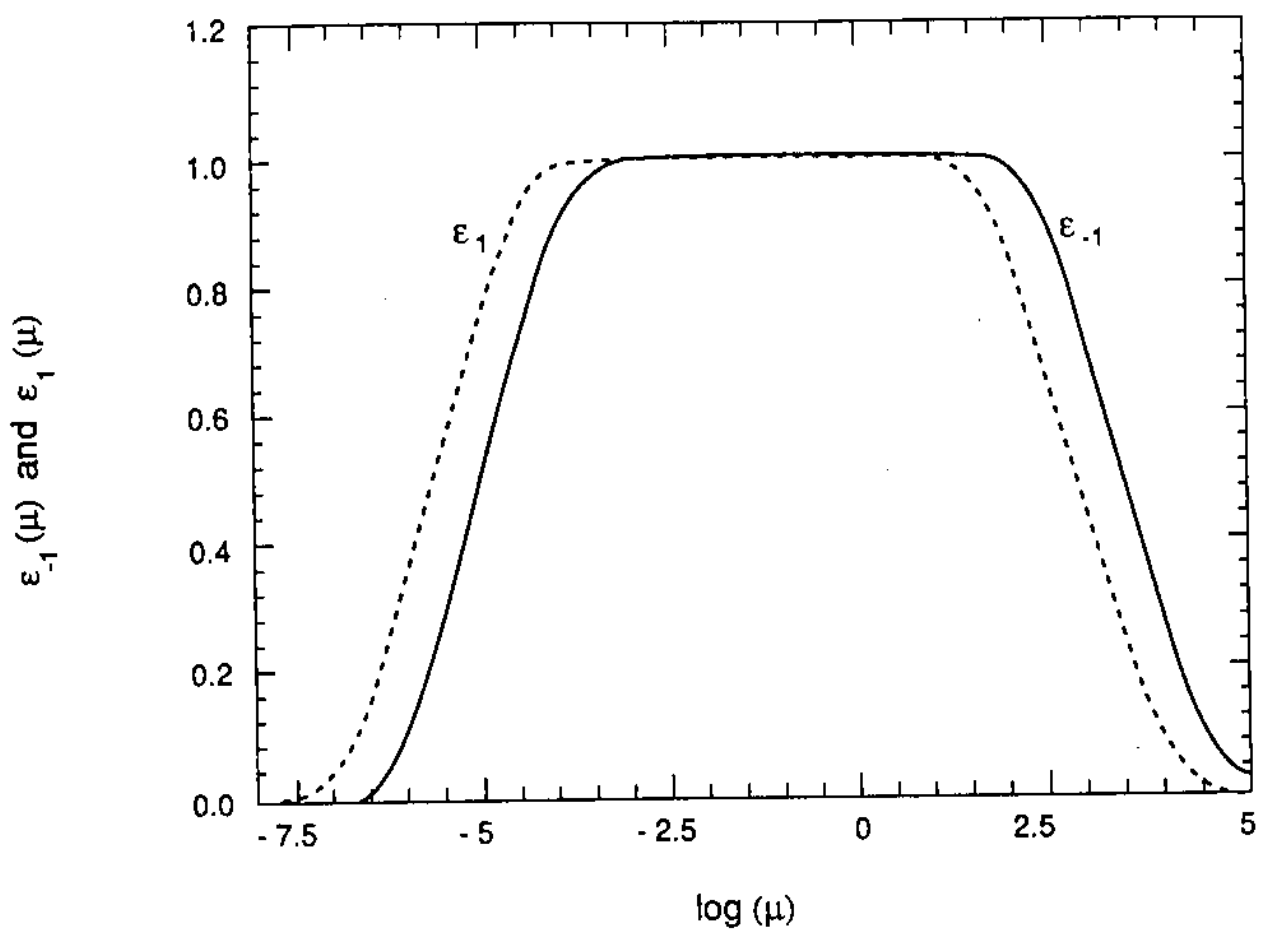

FIGURE 3b

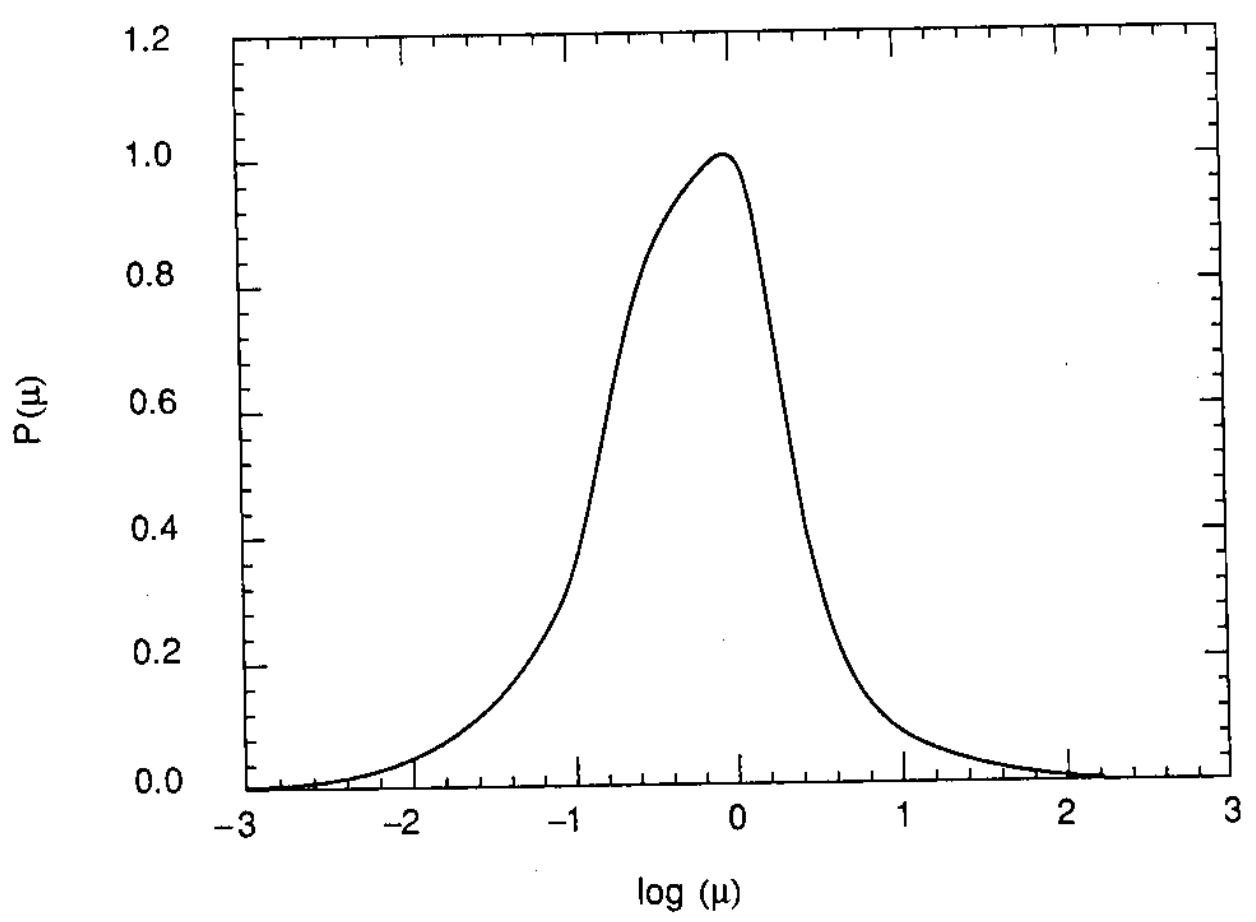

FIGURE 4 


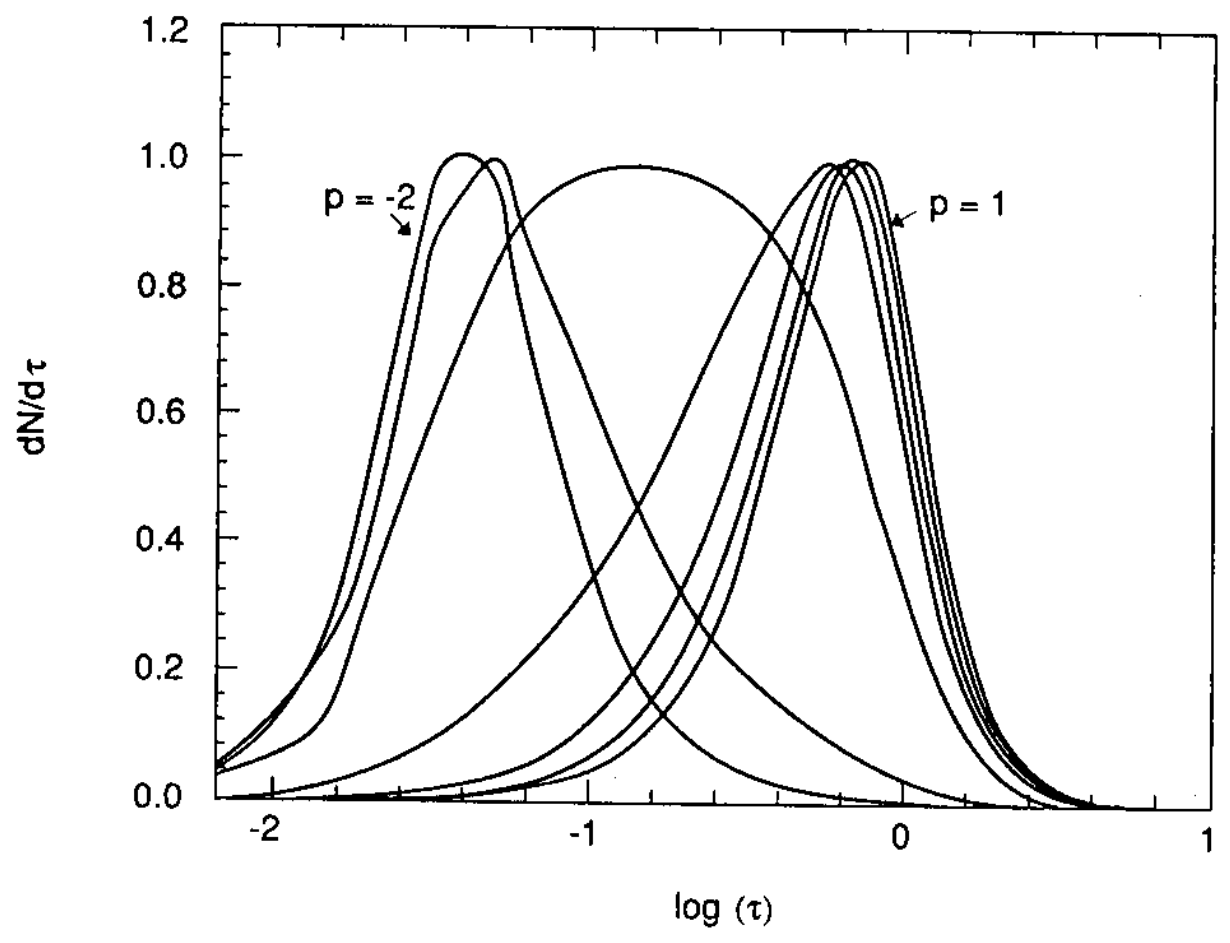

FIGURE 5

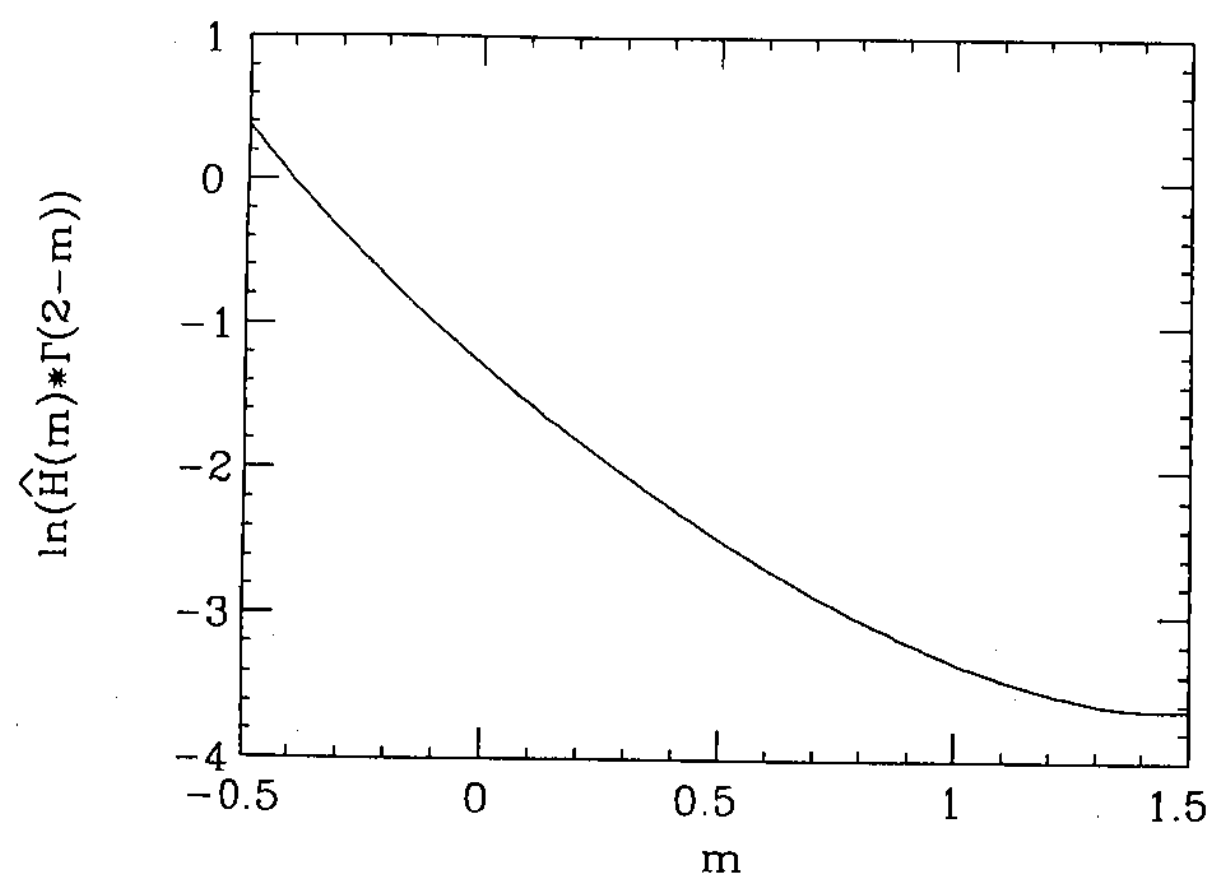

FIGURE 6

Imprimé au CERN. Prix : $0.35 \mathrm{Fr}$. 